\title{
MIGRACIÓN Y ESTRUCTURACIÓN TERRITORIAL DEL ESTADO DE BAJA CALIFORNIA
}

\author{
Por \\ Alba Adriana Wells Ayón*
}

\begin{abstract}
RESUMEN
En este ensayo se considera a Baja California como un producto territorial del fenómeno migratorio, y se realiza una descripción general de la dinámica poblacional que ha experimentado el estado a partir de los constantes movimientos migratorios en destacadas áreas de su espaciogeográfico. Demostrando así la existencia en Baja California de movilidades geográficas de población constituidas principalmente por tres tipos de movimientos: $\mathrm{el}$ interno, el interurbano, y el internacional; además de encontrar la presencia de cadenas de migración interna basadas en sistemas de reciprocidad social. Finalmente, se proporcionan las conclusiones del trabajo.
\end{abstract}

\begin{abstract}
This essay considers Baja California as a territorial product of the migration phenomeron, and makes a general description of the population dynamics which has been experienced from the constant migration movements in important areas of its geographical space. Thus, it demonstrates the existence in Baja California of geographical population movements, in three main types: the internal, the interurban and the international, and also found the existence of internal migration chains based on social reciprocity systems. Finally, the article conclusions are presented.
\end{abstract}

\section{INTRODUCCIÓN}

El crecimiento demográfico de Baja California desde sus inicios, principalmente a partir de la cuarta década de $1900,{ }^{1}$ ha experimentado una compleja dinámica poblacional, caracterizada, sobre todo, por el determinante papel que han jugado los constantes movimientos migratorios en destacadas áreas de su espacio geográfico (CONAPO, 1984:1-12), generado a raíz del incremento de la actividad agrícola, de la demanda creciente de trabajadores obreros para una industria en pleno progreso y de la necesidad de más empleados y profesionistas para el gobierno,

\footnotetext{
* Investigadora de tiempo completo en el Instituto de Investigaciones Sociales de la UABC.

1 Período en que la bonanza agrícola en Baja California, genera en su principio demanda de recursos humanos y se establece una fuerte corriente migratoria del resto del país hacia la entidad (CONAPO-MEXiCO, 1984:7).
} 
comercio y la banca, redundando todo esto en el desarrollo del estado. Este continuo proceso de poblamiento y las transformaciones en las estructuras urbana y socioeconómica por él efectuadas son precisamente los elementos que dan pie a la idea central del presente trabajo, que consiste en identificar a esta entidad como un producto territorial de movilidades humanas espaciales en serie, cuyo trasfondo se traduce en una notable expresión de un eficaz sistema de reciprocidad ${ }^{2}$ tanto social como geográfico, con la consecuente creación de cadenas de movimientos migratorios.

En sí, se reconoce la presencia de dos tipos de movilidades en base a sus implicaciones estructurales y espaciales: por un lado, de tipo social y, por otro, de tipo geográfico. La primera, la movilidad social, origina exclusivamente movimientos verticales de población, mientras que la geográfica, únicamente desplazamientos horizontales; no obstante, se les reconoce a ambas la misma importancia, ya que de su interacción resulta el dinamismo de todo este proceso de cambio.

Este trabajo, se centra específicamente en el tipo de movilidad geográfica (aclarando que no por ello se le resta valor a la social), y describe los tres principales tipos de desplazamientos: migraciones interestatales, migraciones interurbanas, y migraciones internacionales de población. La importancia de esta caracterización radica en obtener un panorama más preciso de la magnitud de la conformación de los movimientos geográficos poblacionales como un reflejo del proceso de integración territorial del estado de Baja California, con la finalidad última de brindar conclusiones que sirvan para señalar lineamientos para el desarrollo de futuros estudios sobre el tema. En este sentido, en su contenido se trata el concepto de movilidad, se caracterizan los diferentes tipos de desplazamientos que componen a la movilidad geográfica, se relaciona la movilidad geográfica con los sistemas de reciprocidad; y por último, se presentan los comentarios finales de este documento.

\section{MOVILIDAD Y ESPACIO}

En su acepción más simple, el concepto de movilidad parece designar solamente un desplazamiento espacial, en este caso, de individuos entre esferas diferentes de un espacio geográfico cualquiera. Sin embargo, dicha noción abarca, además de desplazamientos geográficos (migraciones), movimientos en un espacio más abstracto, delimitado

2 Resulta eficaz porque es un mecanismo que tiende a dar dinamismo a los movimientos espaciales (Jacobs, 1975:140 y Lomnitz, 1977:169). 
por los sectores de actividad, de profesión, de calificación, esto es, desplazamientos sociales (Gaudemar, 1979:14-44).

Ambas movilidades deben ser consideradas como vitales para el desarrollo constante de cualquier estructura espacial, ya que, tal como lo afirma Gaudemar (1979:15-39), los desplazamientos espaciales humanos y las transformaciones del espacio son productos del crecimiento, y los desplazamientos sociales son a su vez productos del papel que realizan los hombres en ese crecimiento.

$\mathrm{La}$ anterior es una de las formas de ver a la movilidad de manera general, ya que su estudio se presenta también de manera particular, refiriéndose exclusivamente al desplazamiento de tipo geográfico; por ejemplo, Zelinsky (1971:61, 219-249) lleva a cabo un trabajo donde proporciona una hipótesis acerca de la transición de la movilidad con la cual argumenta que, en la historia reciente, se ha dado la presencia de patrones definitivos de regularidades en el crecimiento de la movilidad personal a través del binomio espacio-tiempo, y que dichas regularidades al parecer representan un componente esencial del proceso de modernización. A su vez, J.I. Clarke (1984:355), al considerar a la movilidad como un proceso, señala que dicha movilidad de población es un síntoma de su propio dinamismo, de su persistente y continuo cambio; y que su natural aumento (o descenso) puede ser visto como un componente principal de ese cambio, con variaciones en tiempo y espacio, y en diferentes maneras. Es así como la movilidad puede presentar múltiples formas e interrelaciones con las distribuciones y los asentamientos humanos; patrones espaciales en los que se encuentra constantemente envuelta, especialmente en el contexto de una gran diversidad de espacios políticos.

A partir de la noción de movilidad de Gaudemar se presenta una interpretación de este concepto como un continuo proceso en el que se establece el vínculo entre movilidad geográfica y social, en donde los desplazamientos sociales son los que garantizan el reparto y disposición de los individuos - en este caso producto de los desplazamientos geográficos- en las múltiples fases del espacio económico. En las dos últimas nociones expuestas, la movilidad se puede interpretar como un indicador de continuo crecimiento y cambio compensador de desequilibrios espaciales.

De estas perspectivas sobre la movilidad, para fines del presente trabajo, se utilizará una única definición, específicamente la de movilidad geográfica. En este sentido, la movilidad geográfica, o migración, será aquel movimiento geográfico de población que implique un desplazamiento en el espacio territorial que atraviesa cualquier espacio político. 


\section{MOVILIDAD GEOGRÁFICA: INTERREGIONAL, INTERURBANA E INTERNACIONAL}

El fenómeno migratorio sitúa a las regiones en un cspacio geográfico ampliado, diversificado y discontinuo, integrando en este movimiento a dos unidades: el espacio geográfico y el espacio relacional. ${ }^{3}$ Este mecanismo, frecuentemente estudiado por los gégrafos, deja entrever como factor determinante, para el efecto de expulsión o atracción de población, al proceso de modernizacion; lo que hace que el espacio urbano tenga efectos junto con otra serie de determinaciones de carácter histórico, y que marcan los movimientos poblacionales y las transformaciones materiales que producen estos desplazamientos (Ranfla y Álvarez, 1988: 270).

En este sentido, el estado de Baja California se presenta actualmente como una entidad en continuo movimiento, por ser escenario de todo un proceso de desarrollo socioeconómico, pero que debe su configuración territorial a la participación de la dinámica económica y de la dinámica de la población que al mismo tiempo tienen sobre sus espacios.

Dicha dinámica poblacional se manifiesta en forma de dos procesos migratorios: el intemo, que se lleva a cabo entre regiones dentro de los límites del mismo país; y el internacional, entre regiones de un país con otro (CONEPO-UABC, 1987). No obstante, se debe tener presente que la existencia de ambos procesos forman parte de un mismo conjunto de transformaciones a las estructuras territoriales, y que, por lo tanto, no se les puede considerar como procesos separados totalmente, sino interrelacionados en el momento de efectuar su acción sobre el espacio urbanogeográfico del estado.

\section{Migración interregional}

Esta movilidad geográfica, proveniente de diferentes regiones del país hacia Baja California, empieza a cobrar verdadera importancia en las primeras tres décadas de 1900 , originado por el intenso desarrollo que estaban alcanzando las estructuras económicas en la región y que la colocaba como un verdadero punto de atracción para migrantes; ya para la década de los cuarenta, la población había aumentado en 30 veces más su total en comparación con la existente a principios del siglo, pucs de

3 El espacio relacional viene a enriquecer el concepto de la migración, reconociendo en él la existencia de otros factores además del geográfico, como por ejemplo, las relaciones económicas, políticas, sociales y de tiempo que tienen un efecto directo en la dinámica poblacional de una determinada región (Jones, 1982:157). 
alrededor de 7,500 habitantes en 1900 pasó a contar con cerca de 227,000 en 1940, según el censo de 1950 (CONAPO, 1984:5-10). A su vez, de 1900 a 1980 la población había aumentado en 155.3 veces, al registrar el censo de 1980 un total de 1'177,886 habitantes. Comparativamente, este aumento en la proporción de residentes de esta entidad, dentro del total de la población nacional, proporciona un buen indicador del progresivo crecimiento poblacional de la región, ya que de representar el $0.06 \%$ en 1900 , pasa al $1.76 \%$ en 1980 (CONAPO, 1985:15).

Esta dinámica de la migración interestatal, surgida en los cuarenta, coloca a la región entre las principales entidades de México que se distinguen porque se poblaron básicamente por crecimiento social. Aunque en un principio, en reducidos montos de inmigrantes, ya que de contar con un $1.8 \%$ del total nacional en 1940 , pasa a tener cerca de un $6.0 \%$, dos décadas después (Centro de estudios económicos y demográficos, 1981:90).

Todavía a principios de los setenta, la contribución de los movimientos migratorios interestatales a la entidad era de alrededor de una tercera parte de su población total; y para la década de 1970-1980, la distribución porcentual de la población inmigrante en las principales áreas del espacio geográfico era del $45.06 \%$ en Tijuana, $36.50 \%$ en Mexicali, y el $15.41 \%$ y $3.03 \%$ en Ensenada y Tecate. ${ }^{4}$

Existen datos más recientes sobre la dimensión estructural de estos flujos a nivel estatal, obtenidos a través de las encuestas sociodemográficas de población de Baja California aplicadas en los años de 1986, 1988 y en 1989.5 En base a esta información se puede decir que: para el período de 1980-1989 (bien podría ser hasta 1990 si no se presentaron cambios inesperados), sobresale el hecho de la ausencia de diferencias significativas entre los porcentajes de inmigrantes residentes de la entidad arrojados por el censo de 1980 y las estimaciones proporcionadas por las mencionadas encuestas durante los últimos años; a diferencia de décadas anteriores como se puede ver en el cuadro 1 .

En lo que respecta al origen de estos flujos de inmigrantes, se observa que, todavía a principios de los ochenta, se presentaba una composición de la población inmigrante constituida por dos corrientes con diferentes puntos de partida: una que se originaba en entidades vecinas como: Sinaloa, Sonora, y Baja California Sur; y otra cuyo origen se daba en

\footnotetext{
4 A pesar de una disminución manifestada en la contribución de estos movimientos migratorios a la entidad a finales de la década de los sesenta (CONAPO, 1985:224).

Estas encuestas son partedel proyectode investigación sobrelas características sociodemográficas de la población del estado denominado "Actualización demográfica de Baja Califomia", mismas que como parte de un convenio de mutua colaboración se llevaron a cabo por la IIS-UABC Y el CONEPO-BC.
} 
CUADRO 1. Baja California; Distribución porcentual de la población total nativa e inmigrante, 1950-1989 (porcentajes).

\begin{tabular}{lcccc}
\hline Afio & Total & Pob. nativa & Pob. inmigrante & No esp. \\
\hline 1950 & 100.0 & 36.6 & 63.4 & \\
1960 & 100.0 & 38.1 & 61.9 & \\
1970 & 100.0 & 58.4 & 41.6 & \\
1980 & 100.0 & 53.7 & 46.3 & \\
1986 & 100.0 & 52.2 & 41.8 & 6.0 \\
1988 & 100.0 & 56.9 & 43.1 & \\
1989 & 100.0 & 55.8 & 44.2 & \\
\hline
\end{tabular}

FUENTE: Censos de Población de 1950, 1960, 1970, 1980 Encuesta demográfica de Baja California 1986, Encuesta continua de población de Baja California 1988, Encuesta continua de migración de Baja California 1989. CONEPO-BC VIS-UABC.

entidades distantes, principalmente en los estados de Coahuila, Michoacán, Zacatecas y Jalisco (CONAPO, 1984:34). Para finales de los ochenta, los estados de Sinaloa y Sonora continuaban formando parte importante de la primera corriente, mientras que de la segunda, lo seguían siendo las entidades de Jalisco y Michoacán, adicionándose el Distrito Federal (ver cuadro 2). En conjunto, estas dos corrientes representaban, en 1989, más de la mitad de la población inmigrante de la entidad.

CUADRO 2. Baja California: Población total de inmigrantes residentes según lugar de nacimiento, 1986-1989 (porcentajes).

\begin{tabular}{lrrr}
\hline Entidad federat. & 1986 & 1988 & 1989 \\
\hline Jalisco & 17.0 & 16.8 & 17.4 \\
Sinaloa & 11.0 & 10.0 & 14.6 \\
Michoacán & 9.0 & 7.2 & 10.9 \\
Sonora & 8.0 & 12.9 & 8.8 \\
D. F. & 6.0 & 6.6 & 6.7 \\
Otras entidades & 43.0 & 46.5 & 41.6 \\
No esp. & 6.0 & 0.0 & 0.0 \\
Total & 100.0 & 100.0 & 100.0 \\
\hline
\end{tabular}

FUENTE: Encuesta demográfica de Baja California, 1986, (resultados). CONEPOBC/IIS-UABC, Mexicali, B.C., 1987; Encuesta continua de población de Baja California (resultados 1988), CONEPO-BC/IIS-UABC, Mexicali, B.C., 1989; y Encuesta continua de migración de Baja California 1989 (resultados), CONEPO-BC/IIS-UABC, Mexicali, B.C., 1990. 


\section{Migración interurbana}

La información sobre migración que proporcionaron las encuestas efectuadas por el Instituto de Investigaciones Sociales de la Universidad Autónoma de Baja California(IIS-UABC) y el Consejo Estatal de Población de Baja California (CONEPO-BC) en 1986, 1987 y 1989, señala la presencia principalmente de desplazamientos migratorios internos, registrados en la migración de la entidad en esos affos. Para este período, la movilidad espacial de esta población se concentró en emigraciones intramunicipales, intermunicipales y en emigraciones interestatales, con una movilidad geográfica total de $71.9 \%, 80.8 \%$ y $82.0 \%$ de población en cada año; y sobresaliendo de éstos, por la mayor concentración de población, los movimientos intramunicipales y los intermunicipales con un $38.4 \%$, $45.5 \%$ y un $54.0 \%$ respectivamente en esos mismos anos (ver cuadro 3 ).

CUADRO 3. Baja California: Población total de emigrantes según lugar de residencia actual 1986-1989 (porcentajes).

\begin{tabular}{lrrr}
\hline Residencia actual & 1986 & 1987 & 1989 \\
\hline Otra loc. del edo. & 27.5 & 37.3 & 45.4 \\
Otro mpio. del cdo. & 10.9 & 8.2 & 8.6 \\
Otra entidad & 33.5 & 35.3 & 28.0 \\
Estados Unidos & 24.5 & 18.9 & 16.4 \\
Otro país & 2.5 & 0.0 & 0.5 \\
No esp. & 1.1 & 0.3 & 1.1 \\
Total & 100.0 & 100.0 & 100.0 \\
\hline
\end{tabular}

FUENTE: Encuesta demográfica de Baja California, 1986, (resultados), CONEPOBC/IIS-UABC, Mexicali, B.C., 1987; Encuesta continua de migración de Baja California 1987, CONEPO-BC/11S-UABC, Mexicali, B.C.; y Encuesta continua de migración de Baja California 1989 (resultados), CONEPOBC/IIS-UABC, Mexicali, B.C., 1990.

En cuanto a los movimientos espaciales intermunicipales, que son los que más interesan porque son los que representan desplazamientos de mayor significado y se encuentran más de acuerdo al concepto de movilidad geográfica que aquí se maneja, Estrella (1989:51) indica que, a través de los datos que arrojó la encuesta continua de 1987, se puede caracterizar la migración intermunicipal en Baja California; esto a partir de la observación de la distribución de los lugares de residencia actual de quienes fueron declarados como emigrantes de cualquiera de los municipios del 
estado, y que de acuerdo a dicha información resultó que el $77.2 \%$ de la población emigrante se encontraba residiendo en las ciudades de los otros municipios de la entidad y era procedente de alguna de las cuatro localidades urbanas del estado; por lo que se puede suponer que, por lo menos mayoritariamente, se trata del grupo que conforma los flujos migratorios interurbanos. ${ }^{6}$

En relación a este grupo de migrantes, producto específicamente de migraciones interurbanas, se logró observar que de su volumen total acumulado hasta 1987, el $62.6 \%$ se localizaba en la ciudad de Tijuana, el $20.3 \%$ en la ciudad de Ensenada, el $10.1 \%$ en Mexicali y en la ciudad de Tecate el restante $7.0 \%$. Por otro lado, al analizar la distribución de la residencia anterior de dichos migrantes, se destacó el hecho de que, de su total, la mitad proceden de la ciudad de Mexicali. Esta ciudad, como lugar de origen de este tipo de movilidad, se reproduce en las tres ciudades restantes del estado, pues en ellas más de la mitad de los migrantes interurbanos proceden de ella. Ahora bien, en términos de destinos, destaca la capacidad de atracción de la ciudad de Tijuana, pues hacia ella se dirigen al menos dos tercios de todos los migrantes de otros municipios del estado; de esta manera, el $74.9 \%$ de los emigrantes de Ensenada, el $69.6 \%$ de los de Mexicali, y el $73.1 \%$ de los emigrantes de Tecate, tienen como destino la ciudad de Tijuana (Estrella, 1989:51).

Los datos arriba mencionados permiten dimensionar un patrón de movilidad intermunicipal con implícitascaracterísticas interurbanas, sobre todo en los municipios de Mexicali y Tijuana. La presencia de numerosos desplazamientos de población emigrante, en el caso de la ciudad de Mexicali, la colocan como área de expulsión; y a la ciudad de Tijuana como centro de recepción, por los numerosos movimientos espaciales de población inmigrante urbana proveniente de los otros municipios de la entidad.

Este patrón de movilidad se puede entender a partir del comportamiento que ha presentado la estructura económica en la entidad y principalmente en los municipios de Mexicali y Tijuana, ya que, al observar la distribución de la población estatal en los diferentes sectores de actividad de 1950 a 1989, tal y como la presenta el cuadro 4, se pudo encontrar una notable disminución en el primer sector de actividad (agricultura, ganadería y

\footnotetext{
- Este tipo de movilidad geográfica señala los desplazamientos espaciales que ocurren desde áreas estructurales urbanas a urbanas, en este caso, dentro de los límites de una determinada región. No se debe confundir entre movimientos intraurbanos e interurbanos, ya que cuando los movimientos se llevan a cabo dentro de una misma ciudad estos son llamados intraurbanos y cuando los movimientos son efectuados de una ciudad a otra se les denomina interurbanos considerados éstos últimos por Short, como formas dominantes de movimientos urbanos espaciales durante etapas de crecimiento económico de una industrialización madura (Short, J., 1984:65).
} 
pesca) y un marcado incremento en el segundo (industria) y tercero (comercio y servicios), resultando con esto principalmente beneficiada la ciudad de Tijuana, ya que su desarrollo socioeconómico se ha basado en dichos sectores.

CUADRO 4. Baja California: Población económicamente activa por sector de actividad 1950-1989 (porcentajes).

\begin{tabular}{lrrrcr}
\hline Año & Total & Sector I & Sector II & Sector III & N.E. \\
\hline 1950 & 100.0 & 48.5 & 17.3 & 34.2 & 0.0 \\
1960 & 100.0 & 41.6 & 20.2 & 38.2 & 0.0 \\
1970 & 100.0 & 24.0 & 26.8 & 49.2 & 0.0 \\
1980 & 100.0 & 9.5 & 1.4 & 43.3 & 33.0 \\
1989 & 100.0 & 13.3 & 24.6 & 60.7 & 1.4 \\
\hline
\end{tabular}

FUENTE: La información de 1950 a 1980 se obtuvo de: Estudio sociodemográfico del estado Baja California, CONAPO, México, 1984, pp.108-111; y la información para 1989 del reporte de la Encuesta continua de migración de Baja California 1989 (resultados), IIS-UABC/CONEPO-BC, Mexicali, B.C. 1990, p. 21.

Por otra parte, en relación con la distribución por tiempo de residencia del volumen total de migrantes interurbanos, Estrella (1989:54) afirma que, observado a nivel agregado, hasta 1987 el $41.0 \%$ se originó en los últimos diez años; el $37.1 \%$ en el período de 11 a 20 años y el $21.3 \%$ de los migrantes se relocalizaron en los municipios del estado hace más de 20 años (ver cuadro 5).

CUADRO 5. Baja California: Población inmigrante interestatal según tiempo de residencia por municipio, 1987 (porcentajes).

\begin{tabular}{lrrrrr}
\hline \multirow{2}{*}{$\begin{array}{l}\text { Años de } \\
\text { residencia }\end{array}$} & \multicolumn{2}{c}{ Total } & \multicolumn{4}{c}{ Residencia actual } \\
& B.C. & Ensenada & Mexicali & Tecate & Tijuana \\
\hline Total & 100.0 & 20.3 & 10.1 & 7.0 & 62.6 \\
1 a 10 & 41.0 & 8.8 & 6.6 & 4.1 & 21.5 \\
11 a 20 & 37.1 & 6.8 & 1.9 & 2.1 & 26.3 \\
Más de 20 & 21.3 & 4.7 & 1.6 & 0.8 & 14.2 \\
No esp. & 0.6 & 0.0 & 0.0 & 0.0 & 0.6 \\
\hline
\end{tabular}

FUENTE: Encuesta continua de migración de Baja California 1987 (resultados), IIS-UABC/CONEPO-BC, Mexicali, B.C. 


\section{Migración internacional}

En su sentido más amplio, este tipo de movilidad es la que efectúa la población a través de fronteras nacionales. Por lo general, indica dos flujos diferentes: el de individuos que cambian su residencia habitual a otro país, por razones laborales o políticas, y el de personas que trabajan temporalmente (no definitivamente) en otro país (García y Griego, 1988:8). No obstante, es importante destacar la existencia de un tercer flujo, constituido por un grupo de emigrantes que también por cuestiones laborales forman parte de la migración internacional particularmente fronteriza, el de los llamados transmigrantes, considerados como aquellos individuos que de manera cotidiana (no por temporadas) cruzan la frontera sólo para trabajar en el otro país y regresan nuevamente al finalizar sus actividades.

El primer tipo de flujo no representa una proporción significativa dentro del total de población emigrante, al menos en esta entidad, pues en los años de 1986, 1987 y 1989 , representó un $2.5 \%$, un cero porciento, y un $0.5 \%$, respectivamente (ver cuadro 3 ). En cuanto a los flujos constituidos por desplazamientos de individuos cuya razón de migrar de manera internacional radica en cuestiones laborales, se le dedica en este trabajo especial atención por la importancia que han representado sus influencias sobre las transformaciones y estructuración de los espacios geográficos del estado.

Al respecto, se dice que, en general, la tendencia natural de los trabajadores a su relocalización tiene, entre agregados nacionales y regionales, las mismas causas y efectos, en el sentido de que responde a la expansión del proceso de modernización a través de un desplazamiento geográfico de trabajadores que buscan mejores salarios y condiciones de trabajo. Estos movimientos se pueden considerar como mecanismos de ajuste en relación con los diferenciales de salario, y se dan, en sentido estricto, cuando existe una frontera política que separa a dos países, y a su vez son reflejo tanto de dos diferentes estructuras socioeconómicas como de los diferenciales de salario. Lo cual hace pensar en las localidades fronterizas como espacios de regulación de la desigual expansión de los procesos de desarrollo entre sectores y regiones de ambos países. Esta regulación destaca la forma de importación-exportación de trabajadores de manera cíclica, misma que lleva, en función de su dinamismo, a la configuración de desigualdad y formas de regulación en relación a la migración internacional de trabajadores (Ranfla y Álvarez, 1988: 265). Por lo tanto, dichas leyes de desarrollo desigual propician que este tipo de movilidad geográfica se acentúe entre países o regiones con notables diferencias en sus niveles de desarrollo, como ocurre entre México y los Estados Unidos. 
En este sentido, Beatriz Acuña (1988) argumenta que el proceso de la transmigración, tal y como se presenta en la migración internacional entre estos dos países, es un fenómeno contemporáneo de creciente importancia e implicaciones socioeconómicas y políticas; ya que, si bien en un principio la migración en general favoreció la urbanización de la frontera norte de México, la zona fronteriza constituye una importante fuente de mano de obra de la cual forman parte los trabajadores transmigrantes.

Durante affos, los transmigrantes o commuters de la frontera mexicana y canadiense fueron considerados como visitantes de negocios en el país, aún después del establecimiento de cuotas de selección y control para los inmigrantes a los Estados Unidos. No fue sino hasta 1927 que el gobierno de Estados Unidos exigió que todo extranjero que entrara al país debía presentar visa de inmigrante, pero para evitar la obtención de una visa para cada entrada de los trabajadores se ideó una tarjeta de identificación para aquellos extranjeros que cruzaban con frecuencia la frontera.

De esta forma, el llamado transmigrante podía obtener un documento ${ }^{7}$ (tarjeta verde) que le permitía entrar a Estados Unidos en forma cotidiana y, a la vez, lo autorizaba a trabajar en cualquier actividad en ese país. A este respecto, se señala la existencia de tres tipos de transmigrantes legales: los residentes de la frontera mexicana que tienen permiso para trabajar sólo en ciertas zonas y actividades y no para residir de manera permanente en E.U.; los ciudadanos estadounidenses que optan por residir en fronteras mexicanas y pasar a trabajar todos los días a sus empleos en dicho país; y por último, están los llamados commuters o tarjeta verde (Acuña, 1988: 279-284).

Sin embargo, la práctica de la transmigración fronteriza es empleada por algunas personas en forma ilegal, tal es el caso de los white-carders que son residentes de las zonas fronterizas mexicanas y a quienes el gobiemo estadounidense ha extendido una tarjeta de cortesía para visitar cl país y que muchas personas utilizan para cruzar legalmente la frontera y trabajar en forma ilegal dentro de los Estados Unidos.

A partir de la observación de las estimaciones más recientes que se pudieron obtener sobre dichos transmigrantes, al parecer tanto legales como ilegales en Baja California ${ }^{8}$, se logró constatar, en base a la información levantada, la existencia en 1988 de aproximadamente 84,000 personas residentes de esta entidad que declararon que alguna vez habían trabajado en

7 Este documento es la forma I-151 o 551, que tenía la característica de ser de color verde, jo que dio al nombre de green carders o "tarjetas verdes".

La información utilizada aquí, son estimaciones obtenidas a partir de los resultados arrojados por los proyectos de Encuesta continua de población de Baja California 1988 y de Encuesta continua de migración de Baja California 1989. IIS-UABC/CONEPO-BC. 
Estados Unidos o que se encontraban laborando en ese momento. Dicha cifra se ve superada en 1989, presentándose un total estimado de más de 96,000 residentes de esta frontera en la mencionada situación.

Por lo que se refiere al estado de legalidad o ilegalidad laboral de este grupo, para 1988 el $33.3 \%$ de las personas declaró tener tarjeta verde para trabajar en Estados Unidos; el 5.3\% ser ciudadano norteamericano; y el 60.7\% sostuvo haber trabajado o trabajar en Estados Unidos sin formar parte de ninguno de los casos anteriores (dentro de estos últimos se pudiera presentar el trabajo ilegal). Comparativamente con las estimaciones de 1989, el total porcentual de los trabajadores legales se incrementó a un $38.7 \%$ y $6.7 \%$ para cada caso; mientras que, en el caso de los posibles trabajadores ilegales se observa un notable descenso (54.4\%); lo que puede ser reflejo de las recientes reformas a las leyes de inmigración establecidas por el gobierno de los Estados Unidos en este tiempo, esto es la denominada Ley Simpson-Rodino (ver cuadro 6).

\section{CUADRO 6. Baja California: Población total residente de 12 años y más de edad según tenencia de tarjeta verde, estatal, Tijuana y Mexicali 1988-1989 (porcentajes).}

\begin{tabular}{lrrrrrr}
\hline $\begin{array}{l}\text { Tenencia de } \\
\text { tarjeta verde }\end{array}$ & Estatal & $\begin{array}{c}1988 \\
\text { Tijuana }\end{array}$ & Mexicali & Estatal & $\begin{array}{c}1989 \\
\text { Tijuana }\end{array}$ & Mxl. \\
\hline Total & 100.0 & 100.0 & 100.0 & 100.0 & 100.0 & 100.0 \\
Si & 33.3 & 41.4 & 27.0 & 38.7 & 46.5 & 32.6 \\
Cd. americ. & 5.3 & 4.9 & 4.8 & 6.7 & 8.3 & 4.4 \\
No & 60.7 & 52.2 & 68.0 & 54.4 & 45.2 & 62.8 \\
No esp. & 0.7 & 1.5 & 0.2 & 0.2 & 0.0 & 0.2 \\
\hline
\end{tabular}

FUENTE: Encuesta demográfica de Baja California,1986, (resultados), CONEPOBC/IIS-UABC, Mexicali, B.C., 1987; Encuesta continua de población de Baja California (resultados 1988), CONIPO-BC/IIS-UABC, Mexicali, B.C., 1989; y Encuesta continua de migración de Baja California,1989 (resul. tados), CONEPO-BC/IIS-UABC, Mexicaii, B.C., 1990.

En este sentido, las variaciones porcentuales manifestadas a nivel municipal en cuanto a trabajo legal o ilegal de 1988 a 1989, principalmente en Tijuana y en Mexicali, incrementan el grupo que cuenta con tarjeta verde o es ciudadano americano, y disminuye el número de trabajadores que no cuentan con ninguna de estas características (ver cuadro 6). Cabe señalar que este comportamiento se asemeja al que presentan los totales 
estatales observados, por lo que se puede afirmar que precisamente estos dos municipios son los que marcan el patrón de transmigración fronteriza bajacaliforniana, ya que los otros dos municipios del estado (Tecate y Ensenada) presentan cifras casi insignificantes de movimientos de migración internacional. ${ }^{9}$

En cuanto a los puertos de entrada en la frontera mexicana para los transmigrantes legales, hasta 1984 se registró una mayor concentración de trabajadores en las ciudades fronterizas de Tijuana, Ciudad Juárez y Mexicali (Acuña, 1988:284). No obstante, independientemente de la condición de legalidad o ilegalidad de los trabajadores, en lo que respecta a las ciudades de Tijuana y Mexicali, se logró captar de 1988 a 1989, un notable incremento en la cantidad de personas que laboraron o laboraban en Estados Unidos, ya que para 1988 se estimó que en Tijuana había aproximadamente 35,500 personas que declararon haber trabajado o que laboraban en los Estados Unidos, mientras que en 1989 esta cantidad se elevó a más de 40,000 personas. Lo mismo sucede en el caso de la ciudad de Mexicali, ya que de alrededor de 40,000 personas que trabajaban o habían trabajado en Estados Unidos en 1988, se incrementó a un total de poco más de 49,000 personas en 1989.

\section{MOVIMIENTOS GEOGRÁFICOS Y SISTEMAS DE RECIPROCIDAD}

Una vez caracterizados los tres desplazamientos geográficos de población (interregional, interurbanos, e internacional) cuya influencia ha venido tomando parte en el proceso de transformación de los espacios y estructuras urbanas territoriales del estado de Baja California; se puede afirmar que, efectivamente, los movimientos territoriales de población forman parte de todo un proceso dinámico y complejo, ligado a la evolución socioeconómica de un país o región: como en este caso en que el deterioro del sector agropecuario (principal actividad económica del estado hasta 1960) no significó su estancamiento, sino que significó el incremento de la población en el desarrollo de los otros sectores económicos de la entidad. En este sentido, dicho desarrollo socioeconómico viene a ser el reflejo de lo que Jane Jacobs (1975:141-147) reconoce como producto de un eficaz sistema de reciprocidad entre los individuos y los procesos económicos que los envuelven, ya que este sistema funciona en las ciudades no sólo al principio, cuando éstas se forman y crecen, sino

9 De acuerdo a los resultados obtenidos sobre la condición de trabajo en Estados Unidos, por las encuestas continuas de Baja California 1988 y 1989. 
mientras sus economías aumentan y se diversifican sin importar lo complejas que las ciudades lleguen a ser; de esta manera es como se pudiera entender el porqué del estancamiento y desaparición de unas ciudades y la grandeza y crecimiento de otras.

Por otra parte, los desplazamientos geográficos se originan en factores socioeconómicos, tal y como lo afirma Rodolfo Corona, ya que éstos hacen que los individuos se desplacen, generalmente al reconocer la dificultad para solucionar sus carencias o mejorar sus condiciones materiales de vida en los lugares donde habitan, no sin antes contar con la previa identificación de las posibilidades para satisfacer sus privaciones o alcanzar sus expectativas económicas en otros sitios que se las puedan brindar (Corona, 1988:7).

En cuanto al hecho de que los individuos tengan previamente identificadas mejores posibilidades de vida en otro lugar, éste se ha vinculado con patrones de desplazamientos espaciales en forma de cadenas de migración, las cuales perpetúan su dinamismo a través de los canales de comunicación que los migrantes mantienen con amigos o parientes en los lugares de origen (White y Woods, 1980: 44). De esta manera, en el momento en que algunos de los familiares o amistades del migrante optan a su vez migrar a la misma región, se establece un tipo de sistema de reciprocidad, distinto al arriba mencionado, e identificado como un mecanismo de intercambio recíproco entre antiguos y nuevos migrantes, mediante el cual se procede a dar orientación para la vida urbana a los recién llegados, auxilio en su sustento brindándoles alojamiento (si lo requieren), y toda clase de apoyo, ya que este mecanismo es generador de solidaridad (Lomnitz, 1977: 223).

En este sentido, a partir del análisis de información reciente sobre relaciones de parentesco entre los inmigrantes residentes de la entidad producto de movimientos interregionales, ${ }^{18}$ se trató de encontrar los posibles lazos entre migrantes que podrían indicar la existencia de cadenas de desplazamientos migratorios y que brindaría apoyo a la idea de que los flujos de movilidad geográfica en Baja California tomaron parte crucial en su integración, en la transformación de sus espacios territoriales mientras crecía y, sobre todo, como parte vital del complejo proceso de desarrollo de la entidad, no fortuitamente a través del tiempo, sino basados en posibles cadenas de migración autosostenidas por efectivos sistemas de reciprocidad entre migrantes.

\footnotetext{
${ }^{10}$ Los datos se obtuvieron de la Encuesta continua de población de Baja California 1988, y de la Encuesta continua de migración de Baja California 1989; y se procedió a analizar solo a la migración de tipo interregional, por preferir el factor distancia para medir la eficacia de las cadenas de migración en el estado.
} 
El análisis correspondiente, se basa en la población exclusivamente de inmigrantes residentes de la entidad (no nativos), y principalmente en aquellos cuya relación con el jefe del hogar se encuentra fuera del núcleo familiar conyugal (cónyuges e hijos solteros), ya que es en dicha población donde se pudieron encontrar las variaciones más sobresalientes.

De esta forma, a partir del análisis comparativo de la información para el estado, obtenida por las encuestas continuas en 1988 y 1989 , se encontró que, en el caso de relaciones de parentesco de cónyuge o hijos con el jefe del hogar, hablando en números relativos no se presentó ningún aumento sino lo contrario, disminuyó, ya que el resto de la población con lazos de parentesco como: yernos o nueras, nietos, padres, suegros, hermanos, cufiados, otros parientes y otros no parientes del jefe del hogar, sí reportó un incremento en la mayoría de sus componentes en 1989, al igual que en 1988 , los porcentajes más altos en lazos como hermanos o cuñados del jefe del hogar y en otros parientes del jefe.

Además, para este mismo lapso, se captó a nivel estatal una estrecha relación entre el aumento de esta población inmigrante y la coincidencia de los principales lugares de origen de la misma (Jalisco, Sinaloa, Sonora, Michoacán y el D. F.), tal y como se puede observar en los cuadros 7 y 8. Lo cual, en conjunto, parece ser el indicador de la presencia de movimientos migratorios en cadena.

CUADRO 7. Baja California: Distribución porcentual de la población inmigrante residente, según entidad de nacimiento y relación de parentesco con el jefe del hogar (1988).

\begin{tabular}{lrrrrrrr}
\hline Entidad & $\begin{array}{r}\text { Total } \\
\%\end{array}$ & $\begin{array}{r}\text { Yerno } \\
\text { nuera }\end{array}$ & $\begin{array}{c}\text { Nie- } \\
\text { tos }\end{array}$ & $\begin{array}{c}\text { Pa- } \\
\text { dres }\end{array}$ & $\begin{array}{c}\text { Herma- } \\
\text { nos }\end{array}$ & $\begin{array}{r}\text { Otros } \\
\text { parient. }\end{array}$ & $\begin{array}{l}\text { Otros norient. } \\
\text { pariont }\end{array}$ \\
\hline Total & 100.0 & 10.2 & 8.0 & 14.1 & 25.0 & 28.2 & 14.5 \\
Jalisco & 18.9 & 1.6 & 2.4 & 2.7 & 6.2 & 4.8 & 1.2 \\
Sinaloa & 15.1 & 0.8 & 0.2 & 2.4 & 3.3 & 5.5 & 2.9 \\
Sonora & 8.2 & 0.9 & 0.8 & 1.2 & 2.5 & 2.0 & 0.8 \\
Michoacán & 7.1 & 0.5 & 0.3 & 1.6 & 1.1 & 2.9 & 0.7 \\
D. F. & 4.1 & 0.2 & 0.8 & 0.2 & 0.6 & 1.5 & 0.8 \\
Otra entidad & 46.6 & 6.2 & 3.5 & 6.0 & 11.3 & 11.5 & 8.1 \\
\hline
\end{tabular}

FUENTE: Encuesta continua de población de Baja California, 1988 (resultados); Mexicali, Baja California, 1989. Encuesta continua de migración de Baja California 1989 (resultados); Mexicali, Baja Califomia, 1990. 
CUADRO 8. Baja California: Distribución porcentual de la población inmigrante residente, según entidad de nacimiento y relación de parentesco con el jefe del hogar (1989).

\begin{tabular}{|c|c|c|c|c|c|c|c|}
\hline \multirow[b]{2}{*}{ Entidad } & \multicolumn{7}{|c|}{ Relación de parentesco } \\
\hline & $\begin{array}{c}\text { Total } \\
\%\end{array}$ & $\begin{array}{l}\text { Yemo } \\
\text { nuera }\end{array}$ & $\begin{array}{c}\text { Nie- } \\
\text { tos }\end{array}$ & $\begin{array}{l}\text { Pa- } \\
\text { dres }\end{array}$ & $\begin{array}{c}\text { Herma- } \\
\text { nos }\end{array}$ & $\begin{array}{c}\text { Otros } \\
\text { parient. }\end{array}$ & $\begin{array}{l}\text { Otros no } \\
\text { parient. }\end{array}$ \\
\hline Total & 100.0 & 10.4 & 8.7 & 15.9 & 24.0 & 22.2 & 18.8 \\
\hline Jalisco & 16.1 & 0.7 & 2.2 & 4.2 & 2.1 & 5.2 & 1.7 \\
\hline Sinaloa & 14.2 & 1.0 & 0.3 & 1.8 & 3.1 & 4.4 & 3.6 \\
\hline Sonora & 6.5 & 0.9 & 0.8 & 1.3 & 0.9 & 1.7 & 0.9 \\
\hline Michoacán & 12.5 & 3.3 & 1.8 & 2.1 & 2.2 & 1.9 & 1.2 \\
\hline D. F. & 6.2 & 0.4 & 0.8 & 0.4 & 2.2 & 1.3 & 1.1 \\
\hline Otra entidad & 44.5 & 4.1 & 2.8 & 6.1 & 13.5 & 7.7 & 10.3 \\
\hline
\end{tabular}

FUENTE: Encuesta continua de población de Baja California, 1988 (resultados); Mexicali, Baja California, 1989. Encuesta continua de migración de Baja California 1989 (resultados); Mexicali, Baja Califomia, 1990.

Por su parte, a nivel municipal, se puede observar esta misma tendencia, pero con comportamientos diferentes en cada una de las regiones; ya que los inmigrantes internos de los cuatro municipios registran de manera semejante, lugares de nacimiento como: Jalisco, Sinaloa, Sonora y Michoacán; no obstante, en los municipios de Ensenada, Tecate y Tijuana se denota, además, la presencia de originarios del Distrito Federal, mas no asf en Mexicali, el cual, junto con Tijuana son los únicos que cuentan con nativos de Nayarit. Así mismo, únicamente en Ensenada, Tecate y Mexicali por separado sobresalieron porcentajes de inmigrantes provenientes de entidades como Baja California Sur, Durango y Guanajuato, respectivamente (ver cuadro 9).

\section{CONCLUSIONES}

A partir de lo hasta aquí expuesto, se indica la existencia de una movilidad que relaciona tanto desplazamientos sociales de población (verticales), como desplazamientos geográficos de población (horizontales) en Baja California. A este respecto, haciendo a un lado los desplazamientos de tipo vertical con el fin de adoptarlos en futuros estudios de investigación sobre el tema, se tiene que los desplazamientos horizontales de población en esta entidad se distinguen principalmente en base a los siguientes puntos: 
CUADRO 9. Baja California: Distribución porcentual de la población inmigrante residente por municipio, según entidad de nacimiento (1989).

\begin{tabular}{|c|c|c|}
\hline Municipio & Entidad & $\%$ \\
\hline Municipio de Ensenada & $\begin{array}{l}\text { Total } \\
\text { Jalisco } \\
\text { Sinaloa } \\
\text { B.C.S. } \\
\text { Michoacán } \\
\text { Sonora } \\
\text { D. F. } \\
\text { Otro edo. y EE.UU. }\end{array}$ & $\begin{array}{r}100.0 \\
14.0 \\
11.4 \\
10.0 \\
8.5 \\
7.4 \\
5.5 \\
43.0\end{array}$ \\
\hline Municipio de Mexicali & $\begin{array}{l}\text { Total } \\
\text { Sinaloa } \\
\text { Jalisco } \\
\text { Sonora } \\
\text { Guanajuato } \\
\text { Nayarit } \\
\text { Michoacán } \\
\text { Otro edo. y EE.uU. }\end{array}$ & $\begin{array}{r}100.0 \\
18.4 \\
14.7 \\
13.1 \\
9.4 \\
8.5 \\
8.1 \\
28.0\end{array}$ \\
\hline Municipio de Tecate & $\begin{array}{l}\text { Total } \\
\text { Jalisco } \\
\text { Sinaloa } \\
\text { B.C.S. } \\
\text { Sonora } \\
\text { Durango } \\
\text { D. F. } \\
\text { Otro edo. y EE.UU. }\end{array}$ & $\begin{array}{r}100.0 \\
14.9 \\
12.7 \\
12.1 \\
10.5 \\
8.8 \\
7.1 \\
34.0\end{array}$ \\
\hline Municipio de Tijuana & $\begin{array}{l}\text { Total } \\
\text { Jalisco } \\
\text { Michoacán } \\
\text { Sinaloa } \\
\text { D. F. } \\
\text { Nayarit } \\
\text { Sonora } \\
\text { Otro edo. y EE.UU. }\end{array}$ & $\begin{array}{r}100.0 \\
18.5 \\
12.2 \\
11.5 \\
8.8 \\
5.9 \\
5.2 \\
38.0\end{array}$ \\
\hline
\end{tabular}

FUENTE: Encuesta continua de migración de Baja California 1989 (resultados). CONEPO-BC/IIS-UABC. 
a) Se reconocen tres tipos de desplazamientos geográficos u horizontales de población en Baja California: las migraciones interregionales, las migraciones interurbanas y las migraciones internacionales.

b) En lo que se refiere a las migraciones interregionales, sobresale el hecho de que para finales de los ochenta se observó la presencia de dos corrientes de origen de inmigrantes: la primera formada principalmente por los estados de Sinaloa y Sonora y la otra, por Jalisco, Michoacán y el Distrito Federal.

c) Asimismo, para finales de los ochenta, se señaló la presencia de movimientos de emigración sobre todo intramunicipales, intermunicipales e interestatales, acaparando la mayor concentración de población los intramunicipales e intermunicipales. Estos últimos con implícitas características de migraciones interurbanas, principalmente en Tijuana y en Mexicali.

d) En cuanto a la migración internacional, se pueden mencionar tres importantes flujos de población: el de individuos que cambian su residencia a otro país; el de individuos que trabajan temporalmente en otro país; y el de personas que por razones laborales migran, no por temporadas, sino de manera cotidiana a otro país (transmigrantes). Estos dos últimos flujos son los de mayor significado en Baja California.

e) De acuerdo a las estimaciones obtenidas en 1988 y 1989 sobre la relación entre lazos de parentesco y lugar de nacimiento de los inmigrantes residentes del estado, se pudo constatar la existencia de cadenas de migración autosostenidas por efectivos sistemas de reciprocidad entre inmigrantes, manifestándose este mismo comportamiento, aunque con leves variaciones, en cada uno de los municipios de la entidad.

Por último, a partir de la anterior caracterización de las movilidades espaciales geográficas de población, se puede afirmar que las migraciones interregionales, interurbanas e internacionales de población en el estado de Baja California son un reflejo no sólo de factores de origen económico, sino también de factores sociales presentados en forma de una dinámica socioeconómica específica; misma que ha establecido la pauta para el continuo desarrollo y la estructuración territorial de la entidad.

\section{BIBLIOGRAFÍA}

ACUÑA, Beatriz. 1988. "Transmigración legal en la frontera norte de México-Estados Unidos", Revista mexicana de sociologia. Instituto de Investigaciones Sociales de la UNAM, año L, no. 4, octubre-diciembre. 
CENTRO DE ESTUDIOS ECONÓMICOS Y DEMOGRÁFICOS (CEED). 1981. Dinámica de la población de México, Colegio de México, México, 2da. edición.

CLARKE, J. I, 1984. "Mobility, Location and Society". Migration and Mobility, de Boyce, A.J., Taylor \& Francis Ltd., Gran Bretaffa, vol. Xш.

CONEPO-UABC. 1990. Encuesta continua de migración de Baja California 1989 (resultados), Mexicali, Baja California.

CONEPO-UABC. 1989. Encuesta continua de población de Baja California 1988 (resultados). Mexicali, Baja California.

CONEPO-UABC. 1987. Encuesta demográfica de Baja California 1986, (resultados), Mexicali, Baja California.

CONAPO. 1984. Estudio sociodemográfico de Baja California, México. CONAPO. 1985. Baja California demográfico breviario, 1985, México. CORONA Vázquez, Rodolfo. 1988. "Movilidad geográfica: búsqueda de bienestar". Demos, carta demográfica sobre México 1988. Revista de publicación anual. UNAM.

ESTRELLA, Gabriel. 1989. "Migraciones interurbanas en la frontera norte de México", Estudios fronterizos. Instituto de Investigaciones Sociales de la UABC, año VII, vol. IX, no. 20, septiembre-diciembre, Mexicali, Baja California.

GARCÍA Y GRIEGO, Manuel. 1988. "Cifras pequefias, retos grandes", Demos, carta demografica sobre México 1988, de la UNAM y otras importantes instituciones.

GAUDEMAR, Jean Paul de. 1979. Movilidad del trabajo y acumulacion del capital, Ed. Era, México, 1ra. edición en español.

HAUPT, A. 1980. Gufa rápida de población, Population Reference Bureau, Inc. E.U.A., s/e.

JACOBS, Jane. 1975. La economia de las ciudades, Ed. Península, Barcelona, España, 2da. edición.

JONES, J.R. 1982. "Channelization of Undocumental Mexican Migrants to the U.S.", Economic Geography. Vol. 58, no. 2.

LOMNITZ, Larissa. 1977. Cómo sobreviven los marginados, Ed. Siglo XXI, México, 21va. edición.

NACIONES UNIDAS. 1978. "Factores determinantes y consecuencias de las tendencias demográficas", Estudios de población, no. 50, vol. I, Nueva York.

RANFLA, Arturo y Guillermo Álvarez. 1988. "Migración y formas urbanas en el crecimiento de Tijuana: 1900-1984". Revista mexicana de sociologia. Instituto de Investigaciones Sociales de la UNAM, año L, no. 4, octubre-diciembre. 
SHORT, J. 1984. An Introduction to Urban Geography. Routledge and Keagan ed., E.U.A.

SINGER, Paul. 1975. Economla de la urbanización. Ed. Siglo XXI, México. IX CENSO GENERAL DE POBLACIÓN Y VIVIENDA 1970, 1971. Estado de Baja California. SPP-INEGI, México.

WHITE y Woods. 1980. The Geographicals Impacts of Migration. Longman Group, lra. edición.

ZELINSKY, W. 1971. "The Hyphotesis of the Mobility Transition". Geographical Review. 